\title{
A Rare Cause of Obstructive Chronic Hydrocephalus in an Adult Patient: A Case Report of Fourth Ventricle's Foramina Idiopathic Stenosis
}

\author{
Ibrahima Tine ${ }^{1 *}$, Abdou A. Diop ${ }^{1}$, Ababacar Mbengue ${ }^{2}$, Abdou K. Wimenga ${ }^{3}$, Paterne S. Mudekereza ${ }^{3}$, \\ Seydou B. Badiane ${ }^{3}$
}

\author{
${ }^{1}$ Unit of Neurosurgery, Principal Hospital of Dakar, Dakar, Senegal, West Africa \\ ${ }^{2}$ Department of Imaging, Principal Hospital of Dakar, Dakar, Senegal, West Africa \\ ${ }^{3}$ Department of Neurosurgery, University Hospital Center of Fann, Dakar, Senegal, West Africa
}

* Corresponding Author Address: Unit of Neurosurgery; Principal Hospital of Dakar, Nelson Mandela Avenue, B.P 3006, Dakar, Senegal, West Africa. Tel:+221338395050, Fax:+221338395088. E-mail: ibrahimatine78@hotmail.com

Article Type: Case Report

Received: October 28, 2015, Last revised: December 12,2015, Accepted: December 19, 2015

\section{Abstract}

Background \& Importance: Idiopathic stenosis of the foramina of Magendie and Luschka is a rare cause of obstructive hydrocephalus involving the fourth ventricle.

Case Presentation: We reported the case of a 40-year-old woman who developed headaches and vertigo for several months and more recently gait disturbance. The CT scan showed quadri-ventricular hydrocephalus involving mainly the fourth ventricle with dilated lateral recesses. Craniocervical MRI confirmed hydrocephalus and also showed the brainstem and cerebellar tonsil herniation through the foramen magnum with hydromyelia and a hyperintense signal on T2 weighted MRI of cervical spinal cord. Biological analyses were normal. She underwent endoscopic third ventriculostomy (ETV). No complication was observed. The patient became asymptomatic during the weeks following the surgical procedure and remained stable at a mean follow-up interval of 20 months. Postoperative MR images demonstrated regression of the hydrocephalus; complete disappearance of brainstem and cerebellar tonsil herniation, hydromylia and the hyperintense signal on T2 weighted MRI of cervical spinal cord.

Conclusion: This case confirms the existence of hydrocephalus caused by idiopathic fourth ventricle outflows obstruction in adult and the efficacy of ETV for this rare indication.

Keywords: Hydrocephalus; V4 foramina stenosis; ETV

Please cite this paper as: Tine I, Diop A.A, Mbengue A, Wimenga A.K, Mudekereza P.S, Badiane S.B. A Rare Cause of Obstructive Chronic Hydro-cephalus in an Adult Patient: A Case Report of Fourth Ventricle's Foramina Idiopathic Stenosis. Iran. J. Neurosurg. 2015;1(3):26-29.

\section{Introduction}

Idiopathic stenosis of the foramina of Magendie and Luschka is a rare but well-established cause of obstructive hydrocephalus involving the fourth ventricle (5). In children, it is usually the consequence of posterior cerebral fossa (PCF) malformations and in adults, the occlusion is rather acquired than congenital, often linked to an inflammatory process, or sometimes to an infection, head trauma, intraventricular hemorrhage, tumors or Arnold-Chiari malformation (7). It can be treated with a CSF shunt or a suboccipital craniotomy and opening of the membrane obstructing the foramen of Magendie. As neuroendoscopic procedures improved, endoscopic third ventriculostomy (ETV) becomes an excellent option for treatment of these patients because of its efficiency and lower risk than other procedures (3, 6 ). Here we report a case of idiopathic stenosis of the foramina of Magendie and Luschka in an adult patient successfully treated by ETV.

\section{Case Presentation}

We reported the case of a 40-year-old woman with no particular medical history who developed high cranial pressure symptoms like headaches, nausea, photophobia and vertigo for several months and more recently gait disturbance. There was no history of infection or head injury. Physical examination was normal except for gait ataxia. A first CT scan showed quadriventricular hydrocephalus involving mainly the fourth ventricle with dilated lateral recesses (Figure 1). Then a craniocervical MRI was prescribed for more accuracy and confirmed hydrocephalus and also tonsils and brainstem (fourth ventricle) herniation through the foramen magnum. Hydromyelia and transependymal resorption of cerebrospinal fluid (CSF) were shown as a hyperintense signal on T2 weighted MRI of cervical spinal cord (Figure 2). Biological analyses results were normal. She underwent ETV. No complication was observed. The patient became asymptomatic during the weeks following the surgical procedure and remained stable at a mean follow-up interval of 20 months. Postoperative MR images demonstrated regression of the hydrocephalus and complete disappearance of brainstem and cerebellar tonsil herniation, hydromylia and the hyperintense signal on T2 weighted MRI of cervical spinal cord (Figure 3).

\section{Discussion}

Obstruction of the fourth ventricle's exits is a common cause of hydrocephalus. It is usually congenital in children with PCF malformation like Dandy-Walker, Chiari malformation, tuberous sclerosis, spina bifida, platybasia, achondroplasia, basilar impression and atlanto-occipital fusion; but in adults it 

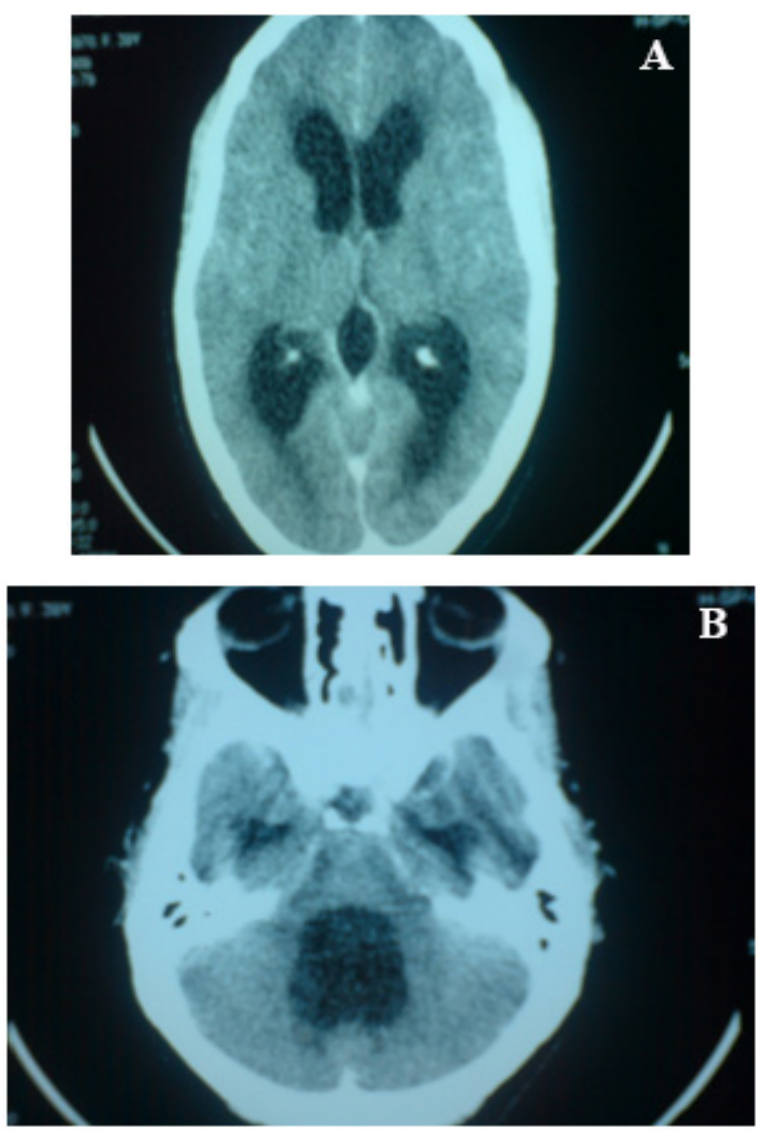

Figure 1. (Pre-operative Images). A: Axial Cerebral CT Scan showing hydrocephalus. B: Axial PCF CT Scan showing enlarged V4

is often linked to an inflammatory process secondary to infection or hemorrhage. In rare cases, no cause is identified $(2,4,5)$. According to Carpentier et al. (1), three different types of obstruction of the foramina of Luschka can occur. In the dysgenesis of the foramen of Luschka, openings in the membrane would lead to a valvular mechanism with intermittent flow of CSF. In the agenesis of the foramen of Luschka, punctiform openings in the membrane would slow the flow of CSF from the ventricular system. In the third type, an arachnoiditis would occur in response to an inflammatory process. There are no typical clinical characteristics. Mainly, signs of compensated intracranial hypertension were reported as headache, nausea and vomiting, papillary edema. Gait ataxia and vertigo were reported as in our case. But acute hydrocephalus with an altered state of consciousness can occur spontaneously or by a minimal head injury $(7,8)$. The diagnosis of hydrocephalus due to fourth ventricle's outlet obstruction can be suspected in case of an imaging study (CT scan or MRI) that shows dilation of all 4 ventricles but mainly involving the forth, and a patent cerebral aqueduct $(3,4,8)$. A significant increase of the lateral recesses with bulging into the cerebellopontine angle may suggest obstruction of the lateral foramina. In Cine-MRI imaging, the CSF flow dynamic can be studied, and help to precisely determine the level of stenosis (8). The absence of cerebrospinal fluid flow from the median outlet may suggest the diagnosis but the CSF flow analysis by MR sequences is less effective at this location than at the cerebral
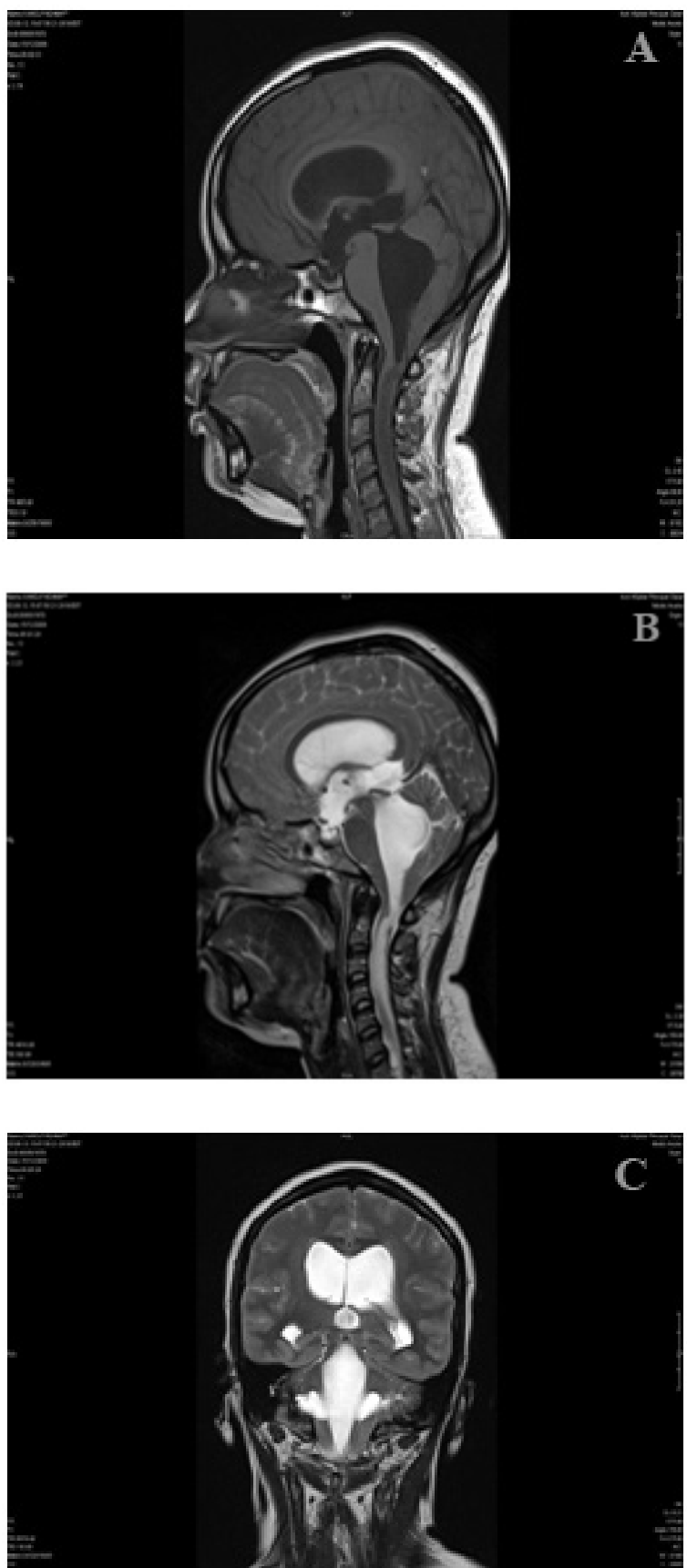

Figure 2. (Pre-operative Images). A: Sagittal T1-weighted image showing a patent aqueduct and enlarged V4. B: Sagittal T2-weighted image showing brainstem and cerebellar tonsil herniation, hydromyelia and CSF transependymal resorption at cervical spinal cord. C: Coronal T2-weighted image showing lateral recess dilation

aqueduct. High resolution MRI may directly visualize the membrane involving the occlusion of the foramen of Magendie as previously reported in a child. However, the membrane was actually visible only on the sagittal T1-weighted MR images (8). On T2-weighted MR images, the membrane was not so evident. Ventriculography was used but it is an invasive method. In our case, MRI also indicated cerebellar tonsils and fourth ventricle herniation through the foramen magnum with normal PCF. Dilation of ependymal central channel with transependymal resorption of CSF mimicking low grade glioma was also noted at the cervical level of spinal cord in our case. This is secondary to the high pressure noted in the fourth ventricle. For the treatment of hydrocephalus due to fourth ventricle's outlet 

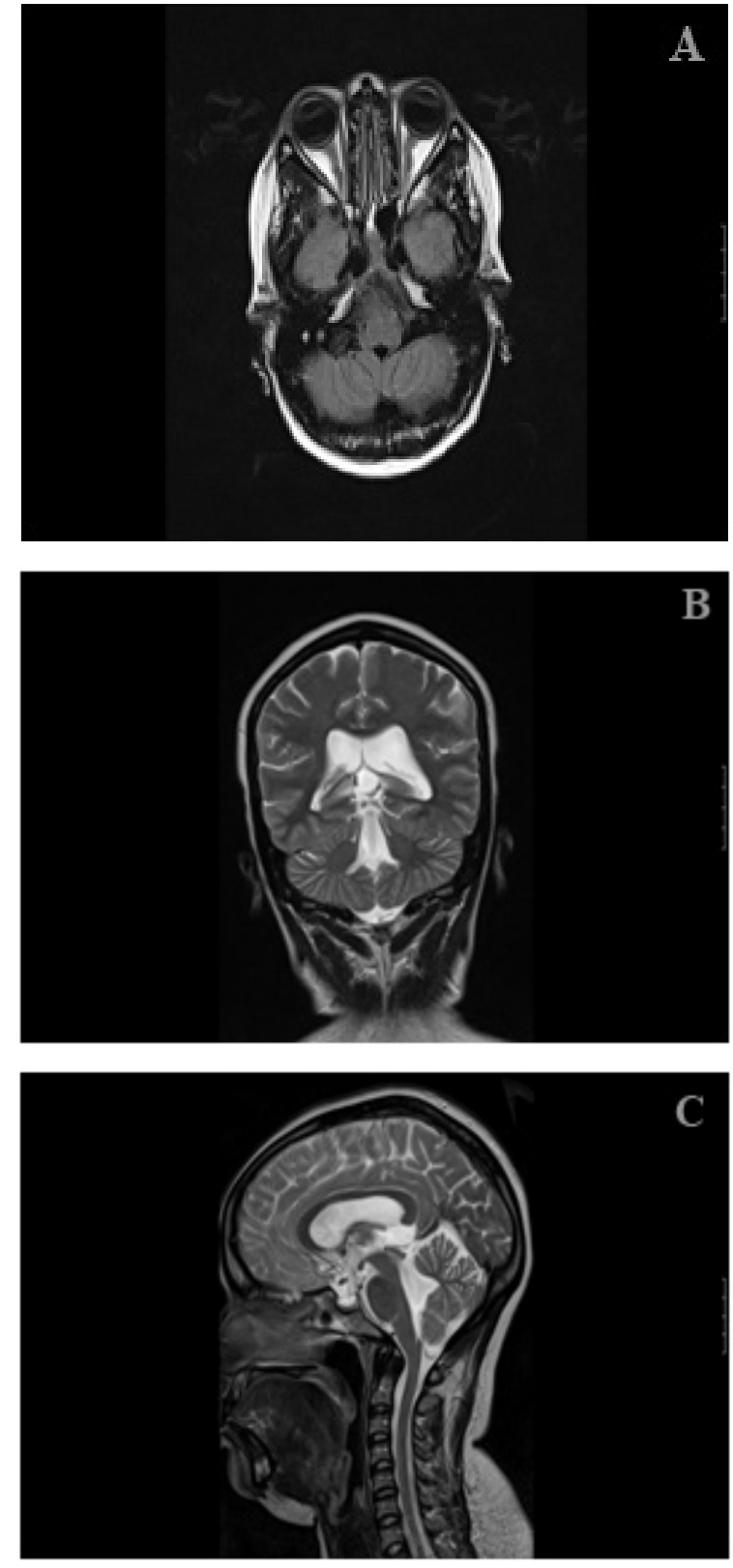

Figure 3. (Post-operative Images): A and B: axial T1-weighted and coronal T2weighted images showing shrinkage of the fourth ventricle and the lateral recess. C: Sagittal T2-weighted image showing complete disappearance of brainstem and cerebellar tonsils herniation, hydromyelia and CSF transependymal resorption

obstruction, various approaches have been described. Older papers suggested the use of CSF shunting (ventriculoperitoneal or ventriculocardiac) or suboccipital craniectomy and opening of the membranes, with or without ventriculocisternal shunt (4). However, CSF shunts are known for their risk of malfunction and infection when suboccipital craniectomy is an invasive procedure $(2,3,7)$. Since the end of 1990 s, the development of endoscopic techniques has led to ETV as the treatment of choice for these patients. In fact, ETV has many advantages; it is a safe physiological procedure and a mini-invasive technique with low risk of infection and low rate of recurrence $(2,7)$. Nevertheless, some cases present with a significant increase of fourth ventricle's volume, which could dislodge the brainstem anteriorly and lead to very small suprasellar and prepontine cisterns like in our case. It may also push the tip of the basilar artery toward the floor of the third ventricle. These distortions may prevent a safe ETV procedure. Minimal dilation of the supratentorial ventricular system can also make ETV procedure difficult (3). Longatti et al. (6) proposed the opening of the foramen of Magendie by transaqueductal endoscopic navigation in fourth ventricle using a flexible endoscope. This technique is not easy and present higher risk of encephalic injury than ETV. Recently, Gianetti et al. (3) proposed a new technique using direct endoscopic approach of the fourth ventricle through a paramedian subocciptal burr hole. This technique can be an alternative for ETV for treatment of hydrocephalus due to obstruction of fourth ventricle's outlets. Idiopathic obstruction of fourth ventricle's foramina is rare. Its diagnosis is facilitated by MRI study. ETV is the safest treatment although there are other effective methods.

\section{Funding}

None.

\section{Conflicts of Interest}

The authors declare that they have no conflicts of interest.

\section{References}

1. Carpentier A, Brunelle F, Philippon J, Clemenceau S: Obstruction of Magendie's and Luschka's foramina. Cine-MRI, aetiology and pathogenesis. Acta Neurochir. 2001; 143:517-522.

2. Fuentes S, Métellus P, Adetchessi T, Dufour H, Grisoli F. [Idiopathic acute obstructive hydrocephalus. Case report]. Neurochirurgie. 2006 Feb;52(1):47-51. ( article in French)

3. Giannetti AV, Malheiros JA, da Silva MC. Fourth ventriculostomy: an alternative treatment for hydrocephalus due to atresia of the Magendie and Luschka foramina. J Neurosurg Pediatr. 2011 Feb;7(2):152-6.

4. Hashish H, Guenot M, Mertens P, Sindou M: [Chronic hydrocephalus in an adult due to congenital membranous occlusion of the apertura mediana ventriculi quartii (foramen of Magendie). Report of two cases and review of the literature.] Neurochirurgie 1999; 45:232-236. (article in French)

5. Karachi C, Le Guérinel C, Brugières P, Melon E, Decq P. Hydrocephalus due to idiopathic stenosis of the foramina of Magendie and Luschka. Report of three cases. J Neurosurg. 2003 Apr;98(4):897-902.

6. Longatti P, Fiorindi A, Feletti A, Baratto V: Endoscopic opening of the foramen of magendie using transaqueductal navigation for membrane obstruction of the fourth ventricle outlets. Technical note. J Neurosurg. 2006; 105:924-927.

7. Mohanty A, Anandh B, Kolluri VR, Praharaj SS: Neuroendoscopic third ventriculostomy in the management of fourth ventricular outlet obstruction. Minim Invasive Neurosurg. 1999; 42:18-21.

8. Rougier A, Ménégon P. MRI evidence of membranous occlusion of the foramen of Magendie Acta Neurochir. 2009; 151:693-694.

\section{Comments}

Reading with interest the manuscript; 'A Rare Cause of Obstructive Chronic Hydrocephalus in an Adult Patient: A Case report of Fourth Ventricle's Foramina Idiopathic Stenosis' which is an informative report for young neurosurgeons, the kind readers may be willing to read the other possible aspects of discussion about such a case in the other words:

1- 'Analysing the presented figures while certainly not all of them are provided, one would like to write a legend like; fig's a, b \& c showing different views of non-contrast enhanced MRI showing four ventricular hydrocephalus with normal size subtentorial posterior fossa volume. The ventricles look to be uniformly dilated, the pulvinar portion and habenular recesses blowed back, the distal end of the acqueduct pushed up and back, the cerebellar 
tonsils indriven into the upper cervical region down to the edge of $\mathrm{C} 2$ (resembling the cases of secondary Chiari malformation) with syrinx formation, the brainstem is pushed forward and smacked to back of the clivus so that the prepeduncular cistern and basilar complex cannot be well identified. The floor of the 3rd. ventricle pushed down to the diaphragm sella changing it to a rather 'empty sella' and no parventricular edema as if the brain has adapted well to the situation!

2- Considering the above descriptions of the images, the case cannot be a proper candidate for ATV. Added to these negative points, the adjusted burr hole to inclinate the sheath of ventriculoscope needs to be located inappropriately behind the coronal suture so that the straight ventriculoscope could pass through the foramen of Monroe and reach a proper place for stomy in the floor of the $3 \mathrm{rd}$. ventricle. 3- Passing through the thinned out region in the floor of the ventricle, one cannot be sure whether he/she can safely enter prepeduncular cistern, transpassing the membrane of Liliquest and maintain a durable gateway for CSF circulation.

4- Interestingly enough, in the T2W lateral, midline MRI provided in fig $2 \mathrm{c}$, this cistern is acceptable open, but one cannot be sure, that is because of good velocity/amount of CSF passing through the subarachnoid space or maintained after collapsing brain and ventricles.

5- I would like to suggest that proper coronal views of postop images be provided to control whether correction of intraventricular CSF circulation has been maintained via the transcortical cannula as the provided gateway, rather than the stomy, as presumed but several researchers working on the physiology of ETV.

6- It may be considered that such a case, getting symptomatic at the age of 40 with signs and symptoms of increased intracranial pressure and not synringomyelia (!!) might be nominated as 'a variant of secondary Chiari malformation treated via a temporary diversional system'.

Abbas Amirjamshidi, MD, MPH, Professor of Neurosurgery, Sina Hospital, Tehran University of Medical Sciences (TUMS). 